\title{
A continuum of dimensional separability
}

\author{
LINDA B. SMITH and M. CATHERINE KILROY \\ Indiana University, Bloomington, Indiana 47401
}

\begin{abstract}
Recent studies of children's perception may be interpreted in terms of a developmental continuum from integrality to separability in the perception of multidimensional variation. Two experiments examined the existence of such a continuum in adult perception. The first experiment indicated that there are dimensional combinations that are neither clearly integral nor separable for adults. Rather, such a combination results in performance patterns intermediate between those found with integral and separable dimensions. The second experiment tested one conceptualization of a continuum of dimensional separability in a speeded classification task. By this conceptualization, the perceived structures of multidimensional variation are orderable by the degree to which the component dimensions are differentiated. Consistent with this view, performances with three-dimensional combinations are shown to be differentially dependent on dimensional and wholistic relations. The importance of the joint consideration of developmental and adult issues in the perception of multidimensional variation is briefly discussed.
\end{abstract}

How is the multidimensional nature of stimulus variation perceived? A growing body of empirical results indicates that the answer to this question depends critically on the specific combination of stimulus dimensions (e.g., Garner, 1970, 1974; Lockhead, 1966, 1972; Monahan \& Lockhead, 1977). Performances with integral combinations, such as saturation and brightness, are independent of the component dimensional relations between stimulus items. The critical relation within an integral stimulus set appears to be wholistic magnitude of difference. In contrast, performances with separable combinations, such as size and brightness, are highly determined by the component dimensional relations and minimally affected by relations of wholistic dissimilarity. Garner (1974) has conceptualized this integrality-separability distinction according to differences in perceived structure. By this notion, wholistic similarity and dimensionally-differentiated relations are objectively available within any multidimensional stimulus set. However, a similarity structure is claimed to be the subjective structure when the dimensions are integral and the dimensional structure is said to be the subjective structure when the dimensions are separable.

Possible explanations of this distinction have been complicated by developmental results. Simply, many dimensional combinations that are separable for adults appear to be integral for children under 8 years

This research was supported in part by a grant from the National Science Foundation (BNS 78-13019) and by a Biomedical Support Grant through Indiana University to the first author. The contribution of Robert Mrzlack in the collection of the data and the comments of Deborah Kemler, James Craig, and Richard Aslin are gratefully acknowledged. of age. Specifically, in the tasks used to define dimensional integrality and separability (see Garner, 1974), young children's performances with adultseparable dimensions pattern in an adult-integral manner (Shepp, 1978; Shepp \& Swartz, 1976; Smith $\&$ Kemler, 1977, 1978). The distinction, then, is not strictly about combinations of stimulus dimensions (cf. Garner, 1974). Further, as these developmental findings have been pursued, it has become clear that the "integrality" of adult-separable dimensions for children is not of exactly the same kind as the integrality of adult-integral dimensions (Kemler \& Smith, 1978, 1979; Smith \& Kemler, 1978). Young children have greater access to the dimensional components of their "integral" variation than do adults. The operational tasks that define "integrality," then, do not converge on one kind of perceived structure.

The two kinds of "integrality" are clarified by considering one speeded classification task that distinguishes between them (Smith \& Kemler, 1978). The structures of two crucial stimulus sets are shown in Figure 1. The rotated set (on the left) conforms to a $45^{\circ}$ rotation of the dimensional set (on the right) in the multidimensional space. Thus, the two required classifications are structurally equivalent under a description of only wholistic relations-that is, under a description in which the experimenter-defined dimensions are undifferentiated from other axes in the space. In contrast, the dimensional structures of the two classificatons are quite different. The partition of the dimensional set is simply organized by value on one dimension while that of the rotated set is complexly organized by values on both dimensions. Smith and Kemler's (1978) question was whether the perceived structure of integral variation was according 

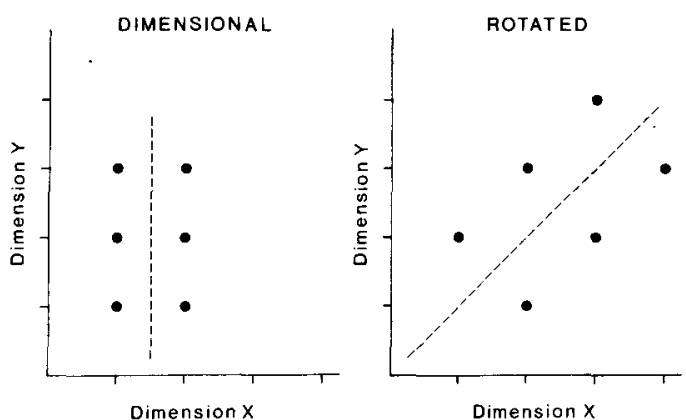

Figure 1. Schematic representations of the structure of the two types of stimulus set (dimensional and rotated) employed in Experiment 1. Each unique item is represented by its component values on the two varying dimensions. The dotted line indicates the required classification in a speeded classification task.

to differentiated (though not completely separate) dimensions. If the perceived structure of the sets is only in terms of wholistic dissimilarity, the dimensional and rotated sets should be sorted equally rapidly. However, if the perceived structure includes the dimensional relations, the dimensional set should be the more rapidly sorted.

Smith and Kemler's (1978) results indicate that adults' perception of the integral dimensions of saturation and brightness are quite wholistic. No advantage of speed sorting the dimensional over the rotated set was observed. (See Lockhead \& King, 1978, for similar results with another adult-integral combination.) However, the dimensions proved to be critical components of the child's "integral" perception of size and brightness, as the dimensional set was sorted much more rapidly than the rotated set. By other indices of integrality, size and brightness are as integral for children as saturation and brightness are for adults (Smith \& Kemler, 1977, 1978). The "integral" perception of children, however, appears to possess some "integral" properties and some "separable" properties. Both wholistic similarity and dimensional relations are critical determiners of the child's performance. Following Lockhead (1972), size and brightness appear to be analyzable for the child: often perceived integrally but also decomposable into the constituent dimensions of change. On the other hand, the integral combination of saturation and brightness seems not to be (at least easily) analyzable for adults.

This finding of "analyzable" and "nonanalyzable" combinations is consistent with Shepard's (1964) notion of a continuum of dimensional separability. Smith and Kemler (1978) hypothesized that there were levels of separability that differed in the subjective primacy of the underlying dimensions-in the degree to which the variation was differentiated into distinct dimensions of change. By this analysis, dimensional combinations are not dichotomized simply according to whether wholistic similarity or dimensional relations dominate. Rather, the perceived structures of multidimensional variation are distinguished by the degree to which the experimenterdefined dimensions organize the apprehension of stimulus differences. If the dimensions are not subjective frames for experience, as saturation and brightness seem not to be (at least for untrained perceivers), the perceived structure can only be one of wholistic magnitudes of difference. In contrast, the separate dimensions, as in the case of size and brightness for adults (Smith \& Kemler, 1978), may totally organize the perception of stimulus difference. Adult integral and separable' combinations are conceptualized as differing in the status (or weight) of component dimensional relations in the perceived structure of variation. In this context, the developmental evidence indicates that the "status" of dimensional relations may fall between " 0 " and " 1. " At such intermediate levels of separability, both wholistic similarity and dimensionally differentiated relations organize the perception of stimulus difference.

The purpose of the present experiment is to examine this hypothesized continuum of dimensional separability in adult perception. This is important because the hypothesized continuum is derived from the intermediate character of children's performances with an adult separable combination. The present position is that developmental level and stimulus dimensions both determine the perceived structure of multidimensional variation (cf. Smith \& Kemler, 1978). By this idea, the "somewhat integral" and "somewhat separable" perception of young children is not simply a degenerate form of adult separability. The prediction is that there are "somewhat integral" and "somewhat separable" combinations in adult perception.

Therefore, the first experiment is directed to finding two dimensions that are perceived neither completely wholistically nor completely separably by adults. The second experiment examines adults' performances with several dimensional combinations. The expectation is that levels of separability will be distinguished by the degree to which performance is determined by the dimensional relations as opposed to the wholistic similarity relations within a stimulus set.

\section{EXPERIMENT 1}

Pilot work suggested length and orientation (of an arrow) to be a good candidate for an intermediate level of separability. Two effects diagnostic of "integrality" in a speeded classification task were examined (cf. Garner, 1974): (1) an interference effect-more rapid sorting when only the "relevant" dimension varies than when both the "relevant" and 
"irrelevant" dimensions vary; and (2) a correlated effect (redundancy gain)-more rapid sorting when the values on the two dimensions are perfectly correlated (and both predictive of the proper response) than when only one dimension varies (and therefore determines the response). An interference effect is important because it indicates that the component dimensions are not immediately perceived as separate sources of information. A correlated effect, on the other hand, suggests an (at least) initial wholistic perception of the differences on both dimensions (see Lockhead, 1972).

The perceptual primacy of length and orientation as distinct dimensions was assessed by comparing the sorting of dimensional and rotated sets (as in Figure 1). More rapid sorting when the classification is structured by the values on one dimension than when structured by perceptually arbitrary axes $45^{\circ}$ from the dimensions implicates the importance of the dimensional relations in the perceived organization. Given comparable wholistic similarity relations within the two stimulus sets, the classification that is better organized by dimensional relations should be perceived as better organized if the experimenterdefined dimensions are primary components of perceived structure.

Thus, if length and orientation are not completely separable, the performance pattern should exhibit some indices of integrality (i.e., an interference effect and/or a correlated effect). If the dimensions are not completely integral, then the dimensional relations within a stimulus set should be critical determiners of performance level (i.e., the dimensional set should be sorted more rapidly than the rotated set).

\section{Method}

Subjects. Sixteen undergraduates at Indiana University participated in partial fulfillment of course requirements.

Stimuli. Arrows varying in length and orientation were mounted on $3 \times 5$ in. white cards.

In the dimensional condition, when length was the relevant dimension for classification, the length values were 1.25 and 1 in.; the potentially irrelevant values of orientation were $20^{\circ}, 40^{\circ}$, and $60^{\circ}$ counterclockwise from horizontal. Set $6 \mathrm{D}$-length contained six unique stimuli: all possible combinations of values on both dimensions. Set 2D-length contained two unique stimuli varying in length only; orientation was held constant at $40^{\circ}$. When orientation was the relevant dimension, its values were $40^{\circ}$ and $60^{\circ}$ and the irrelevant length values were $.75,1.00$, and 1.25 in. Set $6 \mathrm{D}$-orientation contained the items resulting from all combinations of the two dimensions, and the two unique members of set $2 \mathrm{D}$-orientation both possessed the length value of $1 \mathrm{in}$.

In the rotated condition, one stimulus set resulted from combining values such that length was positively correlated with orientation. Thus the members of set $6 \mathrm{R}$ were (in./angle): $1.0 / 20^{\circ}$, $1.25 / 40^{\circ}, 1.50 / 60^{\circ}, .75 / 40^{\circ}, 1.00 / 60^{\circ}$, and $1.25 / 80^{\circ}$. The two unique stimuli in set $2 \mathrm{R}^{\prime}$ were $1.25 / 40^{\circ}$ and $1.00 / 60^{\circ}$. Set $6 \mathrm{R}^{\prime \prime}$ resulted from combining length and orientation values in a negatively correlated manner: $1.25 / 20^{\circ}, 1.00 / 40^{\circ}, .75 / 60^{\circ}, 1.50 / 40^{\circ}$, $1.25 / 60^{\circ}, 1.00 / 80^{\circ}$. The two unique members of set $2 R^{\prime \prime}$ were $1.00 / 40^{\circ}$ and $1.25 / 60^{\circ}$. Note that sets $2 R^{\prime}$ and $2 R^{\prime \prime}$ are the sets with which the correlated effect is measured.
Eight decks (2D-length, 2D-orientation, 6D-length, 6Dorientation, $2 R^{\prime}, 2 R^{\prime}$ ', $6 R^{\prime}$, and $6 R^{\prime \prime}$ ) containing 24 cards were assembled. Unique stimulus items occurred equally of ten within a deck.

Procedure and Design. Each subject speed-sorted four of the eight decks: one $2 D$, one $2 R$, one $6 D$, and one $6 R$. For half the subjects, the classification of the dimensional decks was according to length, for the other half according to orientation. Half the subjects sorting the same dimensional decks also sorted decks $2 \mathbf{R}^{\prime}$ and $6 R^{\prime}$; the other half sorted decks $2 R^{\prime \prime}$ and $6 R^{\prime \prime}$ '. Each trial consisted of speed-sorting the four unique decks (2D, 2R, 6D, 2R). The order of decks within trials was counterbalanced across subjects and reversed on alternate trials. The experimental session consisted of five trials. Prior to sorting each deck, the subject was shown the unique members of the deck and the required classification (as in Figure 1). Exemplars of the set were not in view during speeded sorting. It was emphasized to the subject that speed of sorting (rather than errors) was the critical concern.

\section{Results and Discussion}

Four sorting scores for each subject were calculated by taking the mean of the two fastest sorts of each stimulus set. A preliminary 2 (relevant dimension) by 4 (stimulus set) analysis of variance for a mixed design revealed no reliable effect of relevant dimension $[F(1,14)<1.00]$ or interaction between this factor and stimulus set $[F(3,10)=2.23, p>.10]$. A reliable effect of stimulus set, however, did emerge $[F(3,10)=8.63, p<.01]$. Thus all tests for effects of interest were conducted with scores collapsed across the two levels of relevant dimension. The mean sorting scores and error rates for the four types of stimulus sets are shown in Table 1.

The "integrality" of length and orientation. An interference effect-more rapid sorting of sets $2 \mathrm{D}$ than of sets $6 \mathrm{D}$-was observed $[\mathrm{t}(15)=1.83$, $p<.05]$. However, a reliable correlated effect-more rapid sorting of sets $2 \mathrm{R}$ than of sets $2 \mathrm{D}$-did not obtain $[t(15)<1.00]$. Thus, one effect diagnostic of integrality, interference from variation on an irrelevant dimension but not the other, a redundancy gain, is evident in the present results.

The lack of a correlated effect is somewhat problematic. The effect should occur if subjects' initial processing is wholistic as the (objective) between-group discriminability of sets $2 R$ is greater

Table 1

Mean Sorting Time in Seconds for the Four Types of Stimulus Sets in Experiment 1

\begin{tabular}{cll}
\hline & \multicolumn{2}{c}{ Stimulus Set } \\
\cline { 2 - 3 } Set Size & \multicolumn{1}{c}{ Dimensional (D) } & \multicolumn{1}{c}{ Rotated (R) } \\
\hline 2 & $13.9(.01)^{*}$ & $13.8(.02)$ \\
& {$[0-13.8 ; \mathrm{L}-14.0] \dagger$} & {$\left[\mathrm{R}^{\prime}-14.7 ; \mathrm{R}^{\prime \prime}-12.9\right]$} \\
6 & $15.6(.04)$ & $18.5(.26)$ \\
& {$[0-16.1 ; \mathrm{L}-15.1]$} & {$\left[\mathrm{R}^{\prime}-19.9 ; \mathrm{R}^{\prime \prime}-17.1\right]$} \\
\hline
\end{tabular}

* Mean proportion errors (each subject's two fastest sorts). tMean sorting times in seconds for each unique stimulus deck. 
than that of sets 2D. And the interference effect clearly implicates wholistic processing, at least at some level. Adding a second, correlated, dimension of difference in the case of length and orientation, however, may not always increase the discriminability of two items. Although the specific length and orientation values used were chosen to minimize this possibility, a small illusory effect could have occurred such that lines more closely oriented to vertical appeared longer than the same line more distant from vertical. The present lack of a correlated effect, however, does not seem to be due to such an "illusion." Neither set $2 R^{\prime}$ (positive correlation between length and "verticality" nor set 2R" (negative correlation) is sorted more rapidly than sets $2 \mathrm{D}[\mathrm{t}(7)$ $=-.53$ and $t(7)=1.03$, respectively]. In fact, if there is a trend toward a correlated effect, it occurs with the set $\left(R^{\prime \prime}\right)$ where it is least expected by a verticalhorizontal illusion.

Perhaps at the level of integral and primary dimensions, consistent correlated effects are not to be expected (see Shepp, 1978, for a similar suggestion). The correlated effect has not been uniformly observed when the subjects are young children and the dimensions are separable for adults (Shepp, 1978; Smith \& Kemler, 1978). Two possible reasons for difficulty in obtaining redundancy gains at this intermediate level of experienced dimensionality are: (1) less sensitivity to small differences in wholistic similarity relations when the dimensions are primary than when completely integral, and (2) the relative ease of decomposing the whole into its constituent values (at least when the number of values of each dimension is small, e.g., $\mathbf{n}=2$ ).

The primacy of length and orientation. The superior performance with sets $6 \mathrm{D}$ than with sets $6 \mathrm{R}$ $[t(15)=2.76, p<.01]$ clearly indicates the perceptual primacy of length and orientation as the dimensions that organize stimulus comparisons. The classifications of sets $6 \mathrm{D}$ and $6 \mathrm{R}$ are equally well organized if the experimenter-defined dimensions are not endowed with any special defining status relative to other axes of change (e.g., those $45^{\circ}$ from the dimensions). Subjectively, however, the classifications are not equally well structured. The more rapid sorting of the dimensional set than the rotated set stands in contrast to the earlier results with the integral combination of saturation and brightness (Smith \& Kemler, 1978). Length and orientation while not perceptually immediate (the interference effect) are the reference frames for the perceived structures of the stimulus sets.

In summary, the results of the first experiment suggest that length and orientation are somewhat integral (the interference effect) but also somewhat separable (the rotated effect). This combination for adults, then, is, like size and brightness for young children, intermediate between complete integrality and separability.

\section{EXPERIMENT 2}

This experiment tests the relative importance of the dimensional structure and wholistic similarity structure in adults' perception of three different dimensional combinations. This is done by constructing stimulus sets that differ markedly under a dimensional description and under a wholistic similarity description. Two such stimulus classifications are shown in Figure 2. According to the dimensional relations within each set, the dimensional set is the simpler; in this classification task, a subject need only process the one relevant dimension to sort the stimulus items appropriately. In the similarity task, however, the subject must attend to both varying dimensions; processing of only one or the other leads to response uncertainty for 4 of the 6 items. On the other hand, according to the wholistic-similarity relations within each stimulus set, the similarity task has the advantage over the dimensional task. The within-group similarity is higher and the betweengroup similarity is lower in the similarity classification than in the dimensional one.

Thus, while the stimulus sets employed in Experiment 1 differed greatly only in their dimensional structure, the stimulus sets shown in Figure 2 and used in the present experiment differ in structure, both under a wholistic similarity description and under a dimensional description. And the set that is better organized by the dimensional relations (the dimensional set) is the set least well organized by wholistic similarity. This comparison, then, allows for a direct assessment of the degree of dominance of the dimensions over the wholistic relations within a stimulus set. If the wholistic similarity structure is dominant, the sorting of the similarity set should be the faster of the two. On the other hand, if the dominant relation is one of shared and unshared dimensional values, sorting of the dimensional set should be the more rapid. The degree of difference in speed of sorting the two sets provides an orderable measure of separability. Size-and-brightness sets structured as shown in Figure 2 have been employed in a developmental study (Kemler \& Smith, 1978).

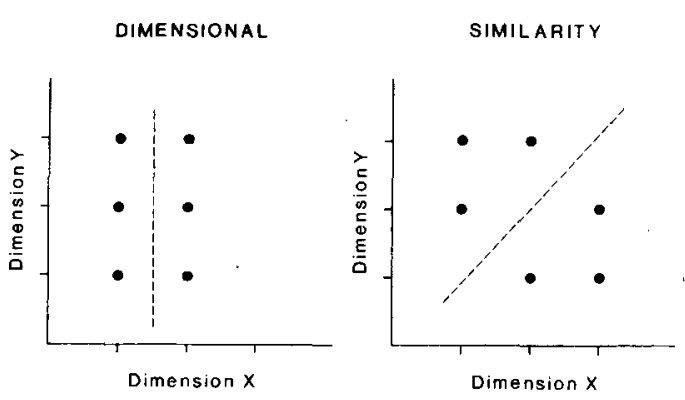

Figure 2. Schematic representations of the structure of the two stimulus sets (dimensional and similarity) employed in Experiment 2. Representation is as in Figure 1. 
The magnitude of the advantage of the dimensional over the similarity set increased with age of perceiver-as expected by the developmental hypothesis of increasing separability. In the present experiment, the magnitude (and direction) of difference between the two sets is expected to be a function of the level of separability of the stimulus dimensions for adults.

The specific stimulus combinations employed are: saturation and brightness - an integral and nonprimary combination by the procedure of Experiment 1 (Smith \& Kemler, 1978); length and orientation-a combination of intermediate separability by the results of Experiment 1; and size and brightness -a separable combination (see Garner, 1974; Smith \& Kemler, 1978). Thus, the predictions: (1) When saturation and brightness vary, the similarity set should be sorted more rapidly than the dimensional set, as these dimensions are hypothesized to be perceived extremely wholistically. (2) When length and orientation vary, marked differences in performance with the two sets are unexpected as both similarity and dimensional relations are important components of the perceived structure of this variation. (3) When size and brightness vary, the dimensional set should be the more rapidly sorted of the two because, at this extreme, the dimensional structure is hypothesized to completely dominate perception.

\section{Method}

Subjects. Twenty-four undergraduates at Indiana University participated in partial fulfillment of course requirements. None had participated in Experiment 1.

Stimuli. The stimulus items were mounted on $3 \times 5$ in. white cards and assembled into decks of 24 cards. The six unique stimuli within each deck occurred equally often.

The size and brightness stimuli were squares with side lengths of three values, $1.00,1.25$, and 1.50 in., cut from Coloraid paper of three shades of gray (Coloraid notation), 1, 2, and 3. The two dimensional decks, structured as shown on the left side of Figure 2, differed by which dimension was relevant for the classification. When size was relevant, its values were 1.25 and 1.00 in.; when brightness was relevant, its values were 1 and 2 . One of the two similarity decks was structured as shown in Figure 2, and the other corresponds to the $90^{\circ}$ rotation of that set in the multidimensional space.

The length and orientation stimuli were constructed as in Experiment 1. The total set consisted of the length values of .75 , 1.00 , and 1.25 in. and the orientation values of $20^{\circ}, 40^{\circ}$, and $60^{\circ}$. Two dimensional and two similarity decks were constructed as above. When length was relevant for classifying the dimensional set, its values were 1.00 and 1.25 in.; when orientation was relevant, its values were $40^{\circ}$ and $60^{\circ}$.

The saturation and brightness stimuli consisted of Munsell chips of constant hue (10R) that varied in chroma and value. The three levels of chroma were 2,4 , and 6 , and the three levels of value were 4,5 , and 6 . The two dimensional sets and the two similarity sets were constructed as above. When chroma was relevant for the classification of a dimensional set, its levels were 4 and 6; when value was relevant, its levels were 4 and 5 .

Procedure and Design. Equal numbers of subjects were assigned
Table 2

Mean Sorting Time in Seconds for Each Stimulus Set and Dimensional Combination in Experiment 2

\begin{tabular}{ccc}
\hline & \multicolumn{2}{c}{ Stimulus Set } \\
\cline { 2 - 3 } Combination & Dimensional & Similarity \\
\hline Saturation-Brightness & 16.3 & 14.7 \\
& $(.16)^{*}$ & $(.08)$ \\
Length-Orientation & 14.1 & 14.5 \\
& $(.03)$ & $(.10)$ \\
Size-Brightness & 12.1 & 15.2 \\
& $(.01)$ & $(.09)$ \\
\hline
\end{tabular}

* Mean proportion errors (each subject's two fastest sorts).

to the three conditions of dimensional combination: size-brightness; length-orientation; saturation-brightness. Each subject speedsorted the four unique decks within each condition (the two unique dimensional decks and the two unique similarity decks).

Prior to speed-sorting each deck, the subject was shown the six unique members of the deck and the required classification. A trial consisted of the speeded sorting of each of the four decks. The order of decks within trials was counterbalanced across subjects in a condition, and was reversed on alternate trials. The session consisted of five trials. The importance of speed of sorting was emphasized to the subjects.

\section{Results and Discussion}

Two sorting scores were calculated for each subject by taking the combined mean of the two fastest sorts of each of the two dimensional sets and the combined mean of the two fastest sorts of each of the two similarity sets. The mean of these dimensional and similarity scores for the three conditions of dimensional combination are shown in Table 2. As can be seen, the predictions about which stimulus set is the better organized for each dimensional combination are confirmed. When the varying dimensions are saturation and brightness-an extremely integral combination-the similarity sets are sorted more rapidly than the dimensional sets $[t(7)=3.18$, $\mathrm{p}<.01]$. When the varying dimensions are the integral but primary combination of length and orientation, however, no difference in speed of sorting the two types of stimulus sets is observed $[t(7)=1.40]$. Finally, when the separable dimensions of size and brightness vary, the dimensional set is sorted more rapidly than the similarity set $[t(7)=2.99, p<.02]$.

This pattern of results provides strong support for a continuum of multidimensional perception. Length and orientation in combination are clearly not as integral or unitary a percept as saturation and brightness or as perceptually separate sources of stimulus difference as size and brightness. The similarity structure of a set determines integral performance, and the dimensional structure of a set determines separable performance. But neither kind of relation completely dominates the perception of length and orientation. 


\section{GENERAL DISCUSSION}

As predicted from the consideration of developmental findings, levels of dimensionally differentiated perception are evident with adult perceivers. Saturation and brightness variation appears to be apprehended in a quite undifferentiated manner. Speeded classification performance is completely determined by the wholistic similarity relations and not by the component dimensional relations. In contrast, the perceived structures of length-and-orientation variation and of size-and-brightness variation include a specification of dimensions of difference. The perception of these latter two combinations, however, differ in the primacy of the component dimensions as the organizers of stimulus difference. The perception of size and brightness variation is completely one of dimensional relations. To predict performance patterns in a speeded classification task with this combination, one need only specify the dimensional relations within the stimulus sets. On the other hand, the dimensional relations appear not to be the total organizers of perceived differences in length and orientation. Both the overall similarity relations and dimensional relations within a stimulus set contribute to performance level.

The distinction between undifferentiated perception and (at least some) dimensional differentiation is highlighted by some recently collected pilot data. Subjects were simply asked to classify pairs of stimuli according to whether the items in the pair differed in "one way," "two ways," or "three ways." Pairs in which the items differed in only saturation, only brightness, or in both saturation and brightness were all classified as differing in "one way" ( $96 \%$ of the time). To the subjects, the differences between pairs were only differences in color. In contrast, these same subjects classified items differing in length and/or orientation and items differing in size and/or brightness according to the number of experimenter-defined dimensions of difference $(84 \%$ and $100 \%$ of the time, respectively). Thus, the dimensions of length and orientation and the dimensions of size and brightness (size and color by the subjects' accounts) are subjective dimensions. The dimensions are experienced as such, and they structure the apprehension of stimulus differences.

It is important to note that the present conceptualization is about the experiential status of dimensions and not about their psychological reality at levels of processing that are not available to the perceiver. For example, if adult subjects are given only saturation and brightness pairs and are told that some differ in "one way" and others in "two ways," they are more likely (though not highly likely) to classify joint differences on both dimensions as a "two-way" difference than differences on only one dimension
(Foard, Note 1). Saturation and brightness, then, may well be the psychological dimensions that underlie the perceived wholistic relation. Phenomenally, however, saturation and brightness are not dimensions on which objects differ to ordinary perceivers. The "integrality-separability" distinction is perhaps primarily a distinction about dimensional combinations at the level of experience. The tasks on which the distinction rests are, for the most part, cognitive tasks (e.g., classification, selective attention).

As noted by Shepard (1964), the contrast between the perception of unanalyzable combinations (saturation and brightness) and analyzable combinations (length-orientation or size-brightness) is an extreme one. Nonetheless, the present work suggests that there are perceptually analyzable combinations of dimensions (i.e., length and orientation) that possess some of the psychological properties of extreme integrality. The existence of this intermediate level of separability is particularly critical for developmental issues. The differences between some dimensional primacy (length-orientation) and complete separability (sizebrightness) in adults - that is, the differences in processing time to perceive the dimensions and in the perceptual importance of overall similarity-are the same kinds of differences that distinguish younger and older subjects' perceptions of (adult) separable dimensions (Shepp, 1978; Shepp \& Swartz, 1976; Smith \& Kemler, 1977, 1978). Thus, the paradoxical indices of both integrality and separability in the child's performance (Kemler \& Smith, 1978, 1979; Smith \& Kemler, 1978) have been partially resolved, here, by showing that there are two levels of integrality in adults- and that one level, like the wholistic perception of the young child, falls between complete integrality and separability.

In fact, two of the formerly "problematic" developmental results-better performance with dimensional than with rotated sets (Smith \& Kemler, 1978) and equivalent performance with dimensional and similarity sets (Kemler \& Smith, 1978)-have been replicated here. However, while this pattern characterizes children's perception of size and brightness, it also characterizes adults' perception of length and orientation. The claim, then, that the perception of multidimensional variation depends on parallel factors of developmental level and stimulus combination remains intact. It is not stimulus factors alone that determine the perceived structure (see Garner, 1974), but rather the interaction between the stimulus and the perceiver (see Monahan \& Lockhead, 1977).

It was specifically a hypothesized link between the integrality-separability distinction and perceptual development that motivated the present work. The combination of size and brightness is completely separable for adults, but only primary, somewhat 
separable, for young children. The expectation is that length and orientation, primary dimensions for adults, will be even less separable, perhaps nonprimary, for young children. The developmental history of most combinations of stimulus dimensions may include stages of quite wholistic perception. In this developmental context, it is reasonable to speculate that the level of separability of particular combinations in adult perception is not fixed. Following Gibson (1969), training and perceptual learning may well increase the separability of adult integral dimensions.

\section{REFERENCE NOTE}

1. Foard, C. Analysis of integral dimensions. Unpublished manuscript, University of Pennsylvania.

\section{REFERENCES}

Garner, W. R. The stimulus in information processing. American Psychologist, 1970, 25, 350-358.

GARNER, W. R. The processing of information and structure. Potomac, Md: Erlbaum, 1974.

Grbson, E. J. Principles of perceptual learning and development. New York: Appleton-Century-Crofts, 1969.

KemLer, D. G., \& SMith, L. B. Is there a developmental trend from integrality to separability in perception? Journal of Experimental Child Psychology, 1978, 26, 498-507.

KEMLER, D. G., \& SMITh, L. B. Accessing dimensional and similarity relations: Adults and young children's discovery of abstract relational concepts. Journal of Experimental Psychology: General, 1979, in press.

LOCKHEAD, G. R. Effects of dimensional redundancy on visual discrimination. Journal of Experimental Psychology, 1966, 72, 95-104.

Lockhead, G. R. Processing dimensional stimuli: A note. Psychological Review, 1972, 79, 410-419.

LoCKhEAD, G. R., \& KING, M. C. Classifying integral stimuli. Journal of Experimental Psychology: Human Perception and Performance, 1978, 3, 436-443.

Monahan, T. S., \& Lockhead, G. R. Identification of integral stimuli. Journal of Experimental Psychology: General, 1977, 106, 94-110.

SHEPARD, R. N. Attention and the metric structure of the stimulus space. Journal of Mathematical Psychology, 1964, 1, 54-87.

SHEPP, B. From perceived similarity to dimensional structure: A new hypothesis about perceptual development. In E. Rosch \& B. Lloyd (Eds.), On the nature and principle of formation of categories. Hillsdale, N.J: Erlbaum, 1978.

Shepr, B. E., \& Swartz, K. B. Selective attention and the processing of integral and nonintegral dimensions: A developmental study. Journal of Experimental Child Psychology, 1976, 22, 73-85.

Smith, L. B., \& Kemler, D. G. Developmental trends in free classification: Evidence for a new conceptualization of perceptual development. Journal of Experimental Child Psychology, 1977, 24, 279-298.

SMITH, L. B., \& KEMLER, D. G. Levels of experienced dimensionality in children and adults. Cognitive Psychology, 1978, 10, $502-532$.

(Received for publication October 24, 1978; revision accepted January $12,1979$. 